\title{
Retrospective Analysis of Route Selection for Hysterectomy for Benign Indications at Ochsner Baptist Hospital
}

\author{
Emily G. Blosser, MD, PhD, ${ }^{1,2}$ George B. Morris IV, MD, ${ }^{1}$ Rajiv B. Gala, MD, FACOG ${ }^{1,2}$ \\ ${ }^{1}$ Department of Obstetrics and Gynecology, Ochsner Clinic Foundation, New Orleans, LA ${ }^{2}$ The University of Queensland Faculty of \\ Medicine, Ochsner Clinical School, New Orleans, LA
}

Background: Hysterectomy, the most common gynecologic procedure in the United States, can be performed in a number of ways. A shift in surgical practice toward cost-effective and minimally invasive approaches provides an impetus to maximize early training in vaginal surgery for resident physicians.

Methods: A total of 62 abdominal, 303 robotic, and 41 vaginal hysterectomies performed between January 1, 2015 and December 31, 2017 at Ochsner Baptist Hospital in New Orleans, LA, that met inclusion criteria were retrospectively reviewed with a previously published route selection algorithm. We applied the algorithm using preoperative and postoperative data collected via medical record review to determine if our practices favor minimally invasive approaches.

Results: Analysis using preoperative variables identified 152 robotic cases that were vaginal hysterectomy candidates (50.2\%). Postoperative analysis of the same cases identified 127 (41.9\%) vaginal hysterectomy candidates. Among abdominal cases, 37 (59.7\%) called for a less invasive approach by preoperative findings: 7 (11.3\%) vaginal and 30 (48.4\%) laparoscopic. The algorithm sorted only 25 of the 62 abdominal cases (40.3\%) to the abdominal approach.

Conclusion: Use of a hysterectomy route selection algorithm preoperatively improves identification of candidates for minimally invasive hysterectomy.

Keywords: Hysterectomy, hysterectomy-vaginal, minimally invasive surgical procedures, robotic surgical procedures

Address correspondence to Rajiv B. Gala, MD, FACOG, Department of Obstetrics and Gynecology, Ochsner Baptist Hospital, 2700 Napoleon Ave., New Orleans, LA 70115. Tel: (504) 842-6680. Email: rgala@ochsner.org

\section{INTRODUCTION}

Hysterectomy, the definitive treatment for many benign gynecologic pathologies, is one of the most common major gynecologic procedures performed in the United States. The different surgical approaches to hysterectomy have unique benefits and risks. Nationally, more than $50 \%$ of hysterectomies are performed through a large abdominal incision. ${ }^{1}$ While the American College of Obstetricians and Gynecologists (ACOG) recommends choosing the least invasive route, the transvaginal approach, a number of patient- and surgeon-specific factors influence route selection. ${ }^{2-6}$

Alternative minimally invasive approaches, such as standard and robotic laparoscopy, have gained attention during the last 2 decades. In 2000, the da Vinci Surgical System (Intuitive Surgical, Inc) became the first robotic surgical platform commercially available in the United States. In 2005, the US Food and Drug Administration approved the da Vinci Surgical System for use in gynecologic surgery. ${ }^{7}$ The robotic system was marketed as a clear improvement to standard laparoscopy in terms of ergonomics, 3-dimensional visualization, and hand- and wrist-like dexterity, and many hospitals nationwide quickly adopted it. ${ }^{2,6}$ Despite its perceived superiority to standard laparoscopy, the da Vinci Surgical System remains cost-prohibitive for many rural and community hospitals, with platform startup carrying a $\$ 1$ to $\$ 2$ million price tag. ${ }^{3,4,8-11}$ Hospital systems that have initiated robotics programs have prioritized robotic surgery to maintain their surgeons' expertise and to improve the overall cost-effectiveness of the device. ${ }^{3,10,12}$ While robotic technology has reduced the number of open hysterectomies by approximately $20 \%$, vaginal hysterectomies have also shown a downward trend overall. ${ }^{13}$

The downward trend in vaginal hysterectomies highlights not only the need for a paradigm shift in surgical planning but also the need for substantial and early training in minimally invasive surgical approaches, such as vaginal surgery. ${ }^{14-17}$ In 2018, the Accreditation Council for Graduate Medical Education (ACGME) revised its minimum numbers for resident education in obstetrics and gynecology to emphasize training in minimally invasive hysterectomy approaches vs laparotomy. ${ }^{18}$ Critical to the success of reprioritizing vaginal hysterectomy is highlighting its feasibility and 
Table 1. Exclusion Criteria

\begin{tabular}{ll}
\hline Adnexal disease as primary indication for surgery & Minimally invasive hysterectomy converted intraoperatively \\
Adnexal torsion & Müllerian or uterine anomalies \\
Age $<18$ years & Ovarian, fallopian tube, or primary peritoneal cancer \\
Cervical cancer more advanced than stage 1A1 & Pelvic kidney \\
$\begin{array}{l}\text { Cesarean hysterectomy } \\
\text { Concomitant anti-incontinence procedures }\end{array}$ & Planned appendectomy, cholecystectomy, or bowel surgery \\
$\begin{array}{l}\text { Concomitant pelvic organ prolapse operation other than pure uterine } \\
\text { Emergent hysterectomy }\end{array}$ & Planned umbilical hernia repair affecting route choice \\
$\begin{array}{l}\text { Endometrial hyperplasia of increased complexity } \\
\text { History of multiple cone excisions with no available cervical tissue }\end{array}$ & Risk-reducing surgery (ie, BRCA positive) \\
Laparoscopic hysterectomy or laparoscopy-assisted vaginal & Supracervical hysterectomy \\
hysterectomy & Tubo-ovarian abscess \\
Mesh-related surgery or excision & Uterine cancer or suspicion for sarcoma \\
\hline
\end{tabular}

cost-effectiveness relative to other approaches by implementing evidence-based algorithms that objectively identify candidates for vaginal surgery. ${ }^{19-21}$ Our hypothesis was that we could identify potentially missed vaginal hysterectomy candidates at Ochsner Baptist Hospital in New Orleans, LA, by using a nationally accepted algorithm.

\section{METHODS}

The study was approved by the Ochsner Institutional Review Board. All authors were on staff at the institution at the time of data collection. The study was designed and executed as a retrospective medical record review of patients who underwent hysterectomy via abdominal, robotic, or vaginal routes for benign indications at Ochsner Baptist Hospital between January 1, 2015 and December 31, 2017. Sixteen general gynecology faculty surgeons were included in the study. Gynecologic oncologists and urogynecologists were excluded.

Laparoscopic-assisted vaginal hysterectomies, supracervical hysterectomies, cesarean hysterectomies, and minimally invasive hysterectomies that had to be converted intraoperatively were excluded. Additional exclusions are listed in Table 1.

Patient demographics, history, physical examination, laboratory and imaging results, operative reports, and postoperative care were manually extracted from electronic health records and entered into an Excel (Microsoft Corp) spreadsheet designed to summarize the perioperative period. Age, body mass index (BMI), surgical indication, parity, significant medical history, history of $>1$ laparotomy, imaging, and physical examination findings were summarized for evaluation of route selection based on preoperative findings. Pathology findings, documented adhesions, length of hospital stay, and postoperative complications and visits were catalogued for evaluation of route selection based on intraoperative and postoperative findings.

The algorithm detailed by Schmitt et $\mathrm{al}^{15}$ was used to retrospectively reclassify the optimal route of hysterectomy based on objective characteristics-preoperative pathology, vaginal accessibility, uterine size, and uterine weight- instead of surgeon preference. We defined vaginal accessibility by a history of $\geq 1$ vaginal delivery and history of $\leq 1$ laparotomy combined with physical examination findings of a uterus $\leq 12$ weeks' gestation size and adequate mobility. Deviations from the expected route were defined as in Schmitt et al. ${ }^{15}$

\section{RESULTS}

During the 3-year study period, 1,077 hysterectomies were performed at Ochsner Baptist Hospital. A total of 194 abdominal cases were reviewed. Of those, 97 were performed by an alternative route or converted, 19 were supracervical hysterectomies, 14 were cesarean hysterectomies, and 2 were radical hysterectomies, leaving 62 abdominal cases available for analysis.

A total of 842 robotic hysterectomies were identified. Of those, 539 cases were excluded for the following reasons: 431 involved gynecologic oncology, 45 were performed by urogynecology, 38 had pelvic masses, 20 were combined procedures, 3 were converted to abdominal or laparoscopic approach, and 2 involved suspected injury related to the Essure device. The remaining 303 robotic cases were included and analyzed.

During the study period, 41 vaginal hysterectomies were performed that met inclusion criteria.

Demographics and baseline characteristics of the patients included in the analysis are summarized in Table 2.

After analysis with preoperative variables including patient BMI, medical history, parity, and history of laparotomy, along with physical examination findings and imaging, the algorithm identified 152 robotic cases that could have been vaginal hysterectomy candidates (50.2\%). When the 303 robotic cases were analyzed with postoperative variables such as intraoperative adhesions and pathology weight and size, 127 (41.9\%) were vaginal hysterectomy candidates.

Among the abdominal cases, the algorithm called for a less invasive approach based on preoperative findings in $37(59.7 \%)$ cases: 7 (11.3\%) vaginal and 30 (48.4\%) laparoscopic based on preoperative findings. Additionally, almost $50 \%$ of cases performed abdominally -30 cases 
Table 2. Demographic Characteristics of Patients by Surgical Approach

\begin{tabular}{lccc}
\hline \multicolumn{1}{c}{ Variable } & & Route of Hysterectomy \\
\cline { 2 - 4 } & Abdominal, $\mathbf{n = 6 2}$ & Robotic, $\mathbf{n = 3 0 3}$ & Vaginal, $\mathbf{n = 4 1}$ \\
\hline Age, years, mean \pm SD & $44.35 \pm 5.88$ & $44.7 \pm 7.75$ & $47 \pm 10.36$ \\
Body mass index, $\mathrm{kg} / \mathrm{m}^{2}$, mean \pm SD & $34.61 \pm 7.34$ & $32.46 \pm 8.5$ & $29.86 \pm 6.74$ \\
Race/ethnicity & & & \\
Black/African American & $56(90.3)$ & $164(54.1)$ & $16(39.0)$ \\
Caucasian & $6(9.7)$ & $130(42.9)$ & $22(53.7)$ \\
Hispanic, nonwhite & - & $5(1.7)$ & $2(4.9)$ \\
Asian & - & $4(1.3)$ & $1(2.4)$ \\
\hline
\end{tabular}

Note: Data are presented as n (\%) unless otherwise indicated.

by preoperative analysis and 27 cases by postoperative analysis-were better laparoscopic candidates. The algorithm sorted only 25 of the 62 abdominal cases $(40.3 \%)$ to the abdominal approach (Table 3).

We sought to verify the algorithm recommendations using postoperative findings. The algorithm overestimated the number of potential vaginal hysterectomy candidates who were better served with a planned robotic approach (152 vs 127 cases, accounting for 25 misclassifications, 8.3\%), primarily because of previously undiagnosed pelvic adhesive disease, endometriosis, or inaccurate estimates of uterine size with physical examination or imaging. In addition, 3 robotic cases and 4 vaginal cases would have been appropriated to the abdominal approach by intraoperative and postoperative findings (7 misclassified cases, 11.3\%).

Conversely, the algorithm at times called for more invasive surgery than was necessary. Sixteen of the 19 robotic cases recommended for an abdominal approach were correctly performed robotically after reviewing postoperative findings.

\section{DISCUSSION}

While the ACOG recognizes 3 acceptable routes of hysterectomy, the College makes no specific or standard recommendations for route selection but instead recommends tailoring route selection to each patient in a way that favors a minimally invasive approach. At Ochsner Baptist Hospital, less invasive routes than the abdominal approach are prioritized. The algorithm shows the greatest utility in the objective selection of a minimally invasive route. Little evidence in the current literature suggests that any minimally invasive approach far outperforms the others in terms of patient recovery, tolerance, and satisfaction. The primary difference between these approaches is thought to be cost. An analysis of cost across all routes of hysterectomy at Ochsner hospitals in Louisiana is ongoing, but we anticipate that implementation of this algorithm and improved route selection will have a significant impact on cost, both to patient and hospital.

Our study demonstrates continued opportunities for optimization of route selection when applied to planned abdominal and robotic hysterectomies. The abdominal cases that could be sorted to a less invasive approach were more likely to sort to a laparoscopic approach than to a vaginal approach (Table 3). However, implementing this algorithm has identified some potential vaginal cases that were missed in favor of an overly invasive abdominal approach. While so few cases are unlikely to drastically alter resident training, they may have a significant impact on those patients' experience with hysterectomy.

As expected, the algorithm identified many new potential vaginal cases. If all of the abdominal and robotic cases that the algorithm identified as potential vaginal cases had been attempted vaginally, the total number of vaginal hysterectomies from January 1, 2015 to December 31, 2017 would have increased from 41 to 171 based on postoperative analysis, with only 2 needing to be converted to a laparoscopic/robotic case (1.2\% of 171). This change would most certainly have a positive impact on resident training in vaginal surgery and would also improve residents' ability to achieve their ACGME minimally invasive hysterectomy numbers more expeditiously. Although it was not done for this study, another important analysis will

Table 3. Booked Surgical Approach vs Route Selection With the Algorithm

\begin{tabular}{lccccccc}
\hline & \multicolumn{3}{c}{$\begin{array}{c}\text { Optimal Route Selection by } \\
\text { Preoperative Analysis }\end{array}$} & & & \multicolumn{2}{c}{$\begin{array}{c}\text { Optimal Route Selection by } \\
\text { Postoperative Analysis }\end{array}$} \\
\cline { 2 - 4 } Booked Surgical Approach & Abdominal & Laparoscopic & Vaginal & & Abdominal & Laparoscopic & Vaginal \\
\hline Abdominal, $\mathrm{n}=62$ & $25(40.3)$ & $30(48.4)$ & $7(11.3)$ & & $32(51.6)$ & $27(43.5)$ & $3(4.8)$ \\
Robotic, $\mathrm{n}=303$ & $19(6.3)$ & $132(43.6)$ & $152(50.2)$ & & $3(1.0)$ & $173(57.1)$ & $127(41.9)$ \\
Vaginal, $\mathrm{n}=41$ & $0(0)$ & $7(17.1)$ & $34(82.9)$ & & $0(0)$ & $2(4.9)$ & $39(95.1)$ \\
\hline
\end{tabular}

Note: Data are presented as n (\%). 
be to examine standard laparoscopy cases for the same 3-year period. We suspect that the algorithm will retrospectively sort a number of additional cases from the laparoscopy pool to the vaginal approach. This review is underway. A prospective approach with standardization of preoperative physical examinations and documentation would also be beneficial in optimizing the algorithm for our hospital system.

One limitation to this study is that the algorithm may overstate the appropriateness of the vaginal approach, primarily because unforeseen intraoperative complications (such as pelvic adhesions, endometriosis, or anatomic abnormalities) may be undiagnosed until the day of surgery. However, the importance of our data showing that many abdominal cases would have sorted to a less invasive approach using the algorithm should be emphasized, as the data suggest that implementing the algorithm might improve patient access to less invasive routes of hysterectomy.

Retrospective medical record review is another limitation. Documentation of preoperative physical examinations was inconsistent. Consequently, potential vaginal cases were possibly overestimated or underestimated, as best judgment was used in the absence of a clear preoperative physical examination. Additionally, morcellation of the uterus made postoperative pathology challenging to assess for size. In such instances, the weight of the specimen was used to evaluate and select an approach, adding subjectivity to the study approach.

Despite these limitations, the study provides compelling evidence that implementation of an algorithm for sorting surgical approaches improves route selection and identifies a greater number of potential vaginal cases.

\section{CONCLUSION}

Use of a hysterectomy route selection algorithm preoperatively can improve identification of candidates for minimally invasive hysterectomy and enhance resident training in vaginal surgery.

\section{ACKNOWLEDGMENTS}

The authors have no financial or proprietary interest in the subject matter of this article.

\section{REFERENCES}

1. Wright JD, Herzog TJ, Tsui J, et al. Nationwide trends in the performance of inpatient hysterectomy in the United States. Obstet Gynecol. 2013 Aug;122(2 Pt 1):233-241.

doi: 10.1097/AOG.0b013e318299a6cf.

2. Aarts JWM, Nieboer TE, Johnson N, et al. Surgical approach to hysterectomy for benign gynaecological disease. Cochrane Database Syst Rev. 2015 Aug 12;2015(8):CD003677. doi: 10.1002/14651858.CD003677.pub5.

3. Dayaratna S, Goldberg J, Harrington C, Leiby BE, McNeil JM. Hospital costs of total vaginal hysterectomy compared with other minimally invasive hysterectomy. Am J Obstet Gynecol. 2014 Feb;210(2):120.e1-120.e6. doi: 10.1016/j.ajog.2013.09.028.

4. Daraï E, Soriano D, Kimata $P$, Laplace C, Lecuru F. Vaginal hysterectomy for enlarged uteri, with or without laparoscopic assistance: randomized study. Obstet Gynecol. 2001 May;97(5 Pt 1):712-126.
5. Price JT, Zimmerman LD, Koelper NC, Sammel MD, Lee S, Butts SF. Social determinants of access to minimally invasive hysterectomy: reevaluating the relationship between race and route of hysterectomy for benign disease. Am J Obstet Gynecol. 2017 Nov;217(5):572.e1-572.e10.

6. Committee on Gynecologic Practice. Committee opinion no 701: choosing the route of hysterectomy for benign disease. Obstet Gynecol. 2017 Jun;129(6):e155-e159. doi: 10.1097/AOG.0000000000002112.

7. Sinha R, Sanjay M, Rupa B, Kumari S. Robotic surgery in gynecology. J Minim Access Surg. 2015 Jan-Mar;1 1 (1):50-59. doi: 10.4103/0972-9941.147690.

8. Sheyn D, Mahajan S, Billow M, Fleary A, Hayashi E, El-Nashar SA. Geographic variance of cost associated with hysterectomy. Obstet Gynecol. 2017 May;129(5):844-853. doi: 10.1097/AOG.0000000000001966.

9. Mittapalli R, Fanning J, Flora R, Fenton BW. Cost-effectiveness analysis of the treatment of large leiomyomas: laparoscopic assisted vaginal hysterectomy versus abdominal hysterectomy. Am J Obstet Gynecol. 2007 May;196(5):e19-e21. doi: 10.1016/j.ajog.2006.12.029.

10. Khorgami Z, Li WT, Jackson TN, Howard CA, Sclabas GM. The cost of robotics: an analysis of the added costs of robotic-assisted versus laparoscopic surgery using the National Inpatient Sample. Surg Endosc. 2019 Jul;33(7):2217-2221. doi: 10.1007/s00464-018-6507-3.

11. Bouquet de Joliniere J, Librino A, Dubuisson JB, et al. Robotic surgery in gynecology. Front Surg. 2016 May 2;3:26. doi: 10.3389/fsurg.2016.00026.

12. Woelk JL, Borah BJ, Trabuco EC, Heien HC, Gebhart JB. Cost differences among robotic, vaginal, and abdominal hysterectomy. Obstet Gynecol. 2014 Feb;123(2 Pt 1):255-262. doi: 10.1097/AOG.0000000000000090.

13. Papalekas E, Fisher J. Trends in route of hysterectomy after the implementation of a comprehensive robotic training program. Minim Invasive Surg. 2018 Sep 17;2018:7362489. doi: $10.1155 / 2018 / 7362489$.

14. Burkett D, Horwitz J, Kennedy V, Murphy D, Graziano S, Kenton $\mathrm{K}$. Assessing current trends in resident hysterectomy training. Female Pelvic Med Reconstr Surg. 2011 Sep;17(5):210-214. doi: 10.1097/SPV.0b013e3182309a22.

15. Schmitt JJ, Carranza Leon DA, Occhino JA, et al. Determining optimal route of hysterectomy for benign indications: clinical decision tree algorithm. Obstet Gynecol. 2017 Jan;129(1):130-138. doi: 10.1097/AOG.0000000000001756.

16. Occhino JA, Gebhart JB. Difficult vaginal hysterectomy. Clin Obstet Gynecol. 2010 Mar;53(1):40-50. doi: 10.1097/GRF.0b013e3181 ce8945.

17. Figueiredo O, Figueiredo EG, Figueiredo PG, Pelosi MA III, Pelosi MA. Vaginal removal of the benign nonprolapsed uterus: experience with 300 consecutive operations. Obstet Gynecol. 1999 Sep;94(3):348-351.

18. Review Committee for Obstetrics and Gynecology. Case log minimum numbers: obstetrics and gynecology. Accreditation Council for Graduate Medical Education. https://acgme.org/ Portals/0/PFAssets/ProgramResources/220_Ob_Gyn_ Minimum_Numbers_Announcement.pdf?ver=2020-07-10145129-077. Updated July 2020. Accessed October 9, 2020.

19. Moen M, Walter A, Harmanli O, et al.; Society of Gynecologic Surgeons Education Committee. Considerations to improve the evidence-based use of vaginal hysterectomy in benign gynecology. Obstet Gynecol. 2014 Sep;124(3):585-588. doi: 10.1097/AOG.0000000000000398. 
20. Kovac SR, Barhan S, Lister M, Tucker L, Bishop M, Das A. Guidelines for the selection of the route of hysterectomy: application in a resident clinic population. Am J Obstet Gynecol. 2002 Dec;187(6):1521-1527. doi: 10.1067/mob.2002.129165.
21. Kovac SR. Route of hysterectomy: an evidence-based approach. Clin Obstet Gynecol. 2014 Mar;57(1):58-71. doi: $10.1097 /$ GRF.0000000000000009.

This article meets the Accreditation Council for Graduate Medical Education and the American Board of Medical Specialties Maintenance of Certification competencies for Patient Care, Medical Knowledge, and Practice-Based Learning and Improvement.

(C2020 by the author(s); licensee Ochsner Journal, Ochsner Clinic Foundation, New Orleans, LA. This article is an open (c) (i) access article distributed under the terms and conditions of the Creative Commons Attribution (CC BY) license (creativecommons.org/licenses/by/4.0/legalcode) that permits unrestricted use, distribution, and reproduction in any medium, provided the original author(s) and source are credited. 